\title{
Modification of Lesnic's Approach and New Analytic Solutions for Some Nonlinear Second-Order Boundary Value Problems with Dirichlet Boundary Conditions
}

\author{
Abdelhalim Ebaid \\ Department of Mathematics, Faculty of Science, Tabuk University, P. O. Box 741, Tabuk 71491, \\ Saudi Arabia \\ Reprint requests to A. E.; E-mail: halimgamil@yahoo.com
}

Z. Naturforsch. 65a, 692 - 696 (2010); received August 24, 2009 / revised December 17, 2009

For solving nonlinear boundary value problems (BVPs), a main difficulty of using Adomian's method is to find a canonical form which takes into account all the boundary conditions of the problem. This difficulty is overcome by using a modification for Lesnic's approach developed in this paper. The effectiveness of the proposed procedure is verified by two nonlinear problems: the nonlinear oscillator equation and Troesch's problem.

Key words: Adomian Decomposition Method; Nonlinear Oscillator; Troesch's Problem.

\section{Introduction}

Over the last 25 years, the Adomian decomposition method [1-9] has been shown to solve effectively and easily a large class of linear and nonlinear ordinary and partial differential equations. The main advantage of the method is that it can be used directly without using restrictive assumptions or linearization. The convergence of the method was established by several authors [10-13]. Also, the method has been used by several authors [14-27] to investigate various boundary value problems. Moreover, several problems which have nice applications in science and engineering were solved via Adomian's method by the authors [28-36]. In this paper, we aim to develop the work introduced by Lesnic and Elliott in [37] and use it to solve some nonlinear boundary value problems. It might seem reasonable, before launching into the main idea of this paper, to present a brief outline of Lesnic's approach.

\section{Lesnic's Approach}

In [37], Lesnic and Elliott proposed the operator

$$
\begin{aligned}
& L_{x x}^{-1}(.)= \\
& \int_{x_{0}}^{x} \mathrm{~d} x^{\prime} \int_{x_{0}}^{x^{\prime}}(.) \mathrm{d} x^{\prime \prime}-\frac{x-x_{0}}{1-x_{0}} \int_{x_{0}}^{1} \mathrm{~d} x^{\prime} \int_{x_{0}}^{x^{\prime}}(.) \mathrm{d} x^{\prime \prime}
\end{aligned}
$$

to solve the Dirichlet problem for the heat equation $u_{t}=u_{x x}, 0<x<1, t>0$, under the boundary con- ditions

$$
u\left(x_{0}, t\right)=h(t), \quad u(1, t)=f(t),
$$

without any illustration or derivation. Using this definition, we note that

$$
\begin{aligned}
L_{x x}^{-1}\left(u_{x x}\right)= & u(x, t)-u\left(x_{0}, t\right) \\
& -\frac{x-x_{0}}{1-x_{0}}\left[u(1, t)-u\left(x_{0}, t\right)\right],
\end{aligned}
$$

i. e., the boundary conditions can be used directly to solve the heat equation in $x$-direction. However, from (1) we note that the lower bound of all integrations is restricted to the initial point $x_{0}$. In fact, we can avoid this restriction by using a new definition of $L_{x x}^{-1}$, which gives the same result as in (3), given by

$$
\begin{aligned}
L_{x x}^{-1}(.)= & \int_{x_{0}}^{x} \mathrm{~d} x^{\prime} \int_{c}^{x^{\prime}}(.) \mathrm{d} x^{\prime \prime} \\
& -\frac{x-x_{0}}{1-x_{0}} \int_{x_{0}}^{1} \mathrm{~d} x^{\prime} \int_{c}^{x^{\prime}}(.) \mathrm{d} x^{\prime \prime},
\end{aligned}
$$

where $c$ is the free lower point. This free point plays an important role if the equation being solved has a singular point. Consequently, we propose the following operator to solve second-order Dirichlet BVPs:

$$
\begin{aligned}
L_{x x}^{-1}(.)= & \int_{a}^{x} \mathrm{~d} x^{\prime} \int_{c}^{x^{\prime}}(.) \mathrm{d} x^{\prime \prime} \\
& -\frac{x-a}{b-a} \int_{a}^{b} \mathrm{~d} x^{\prime} \int_{c}^{x^{\prime}}(.) \mathrm{d} x^{\prime \prime}, a \neq b,
\end{aligned}
$$

$c$ is arbitrary. 
In the next section, we introduce a theoretical derivation of the operator given by (5).

\section{Derivation of the Proposed Operator}

Suppose that

$$
u(a)=\alpha \quad \text { and } \quad u(b)=\beta
$$

are the boundary conditions of a second-order ordinary differential equation. Firstly, we define $L_{x x}^{-1}$ as

$$
\begin{aligned}
& L_{x x}^{-1}(.)= \\
& \int_{a}^{x} \mathrm{~d} x^{\prime} \int_{c}^{x^{\prime}}(.) \mathrm{d} x^{\prime \prime}-f(x) \int_{d}^{b} \mathrm{~d} x^{\prime} \int_{e}^{x^{\prime}}(.) \mathrm{d} x^{\prime \prime},
\end{aligned}
$$

where $f(x)$ is to be determined such that $L_{x x}^{-1}\left(u^{\prime \prime}(x)\right)$ can be expressed only in terms of the boundary conditions given in (6). With this definition, we obtain

$$
\begin{gathered}
L_{x x}^{-1}\left(u^{\prime \prime}(x)\right)=u(x)-u(a)-(x-a) u^{\prime}(c) \\
-f(x)\left[u(b)-u(d)-(b-d) u^{\prime}(e)\right] \\
=u(x)-u(a)-f(x)[u(b)-u(d)] \\
-(x-a) u^{\prime}(c)+f(x)\left[(b-d) u^{\prime}(e)\right] .
\end{gathered}
$$

Setting $d=a$ and $e=c$, therefore

$$
\begin{aligned}
& L_{x x}^{-1}\left(u^{\prime \prime}(x)\right)=u(x)-u(a)-f(x)[u(b)-u(a)] \\
& \quad-(x-a) u^{\prime}(c)+f(x)\left[(b-a) u^{\prime}(c)\right] .
\end{aligned}
$$

In order to express $L_{x x}^{-1}\left(u^{\prime \prime}(x)\right)$ in terms of the two boundary conditions only, we have to eliminate the condition $u^{\prime}(c)$ by setting

$$
-(x-a) u^{\prime}(c)+f(x)\left[(b-a) u^{\prime}(c)\right]=0 .
$$

Solving this equation for $f(x)$, assuming that $u^{\prime}(c) \neq 0$, we get

$$
f(x)=\frac{x-a}{b-a} .
$$

Substituting (11) into (7) and (9), respectively, we obtain

$$
\begin{aligned}
& L_{x x}^{-1}\left(u^{\prime \prime}(x)\right)= \\
& u(x)-u(a)-\frac{x-a}{b-a}[u(b)-u(a)]
\end{aligned}
$$

and

$$
\begin{aligned}
& L_{x x}^{-1}(.)= \\
& \int_{a}^{x} \mathrm{~d} x^{\prime} \int_{c}^{x^{\prime}}(.) \mathrm{d} x^{\prime \prime}-\frac{x-a}{b-a} \int_{a}^{b} \mathrm{~d} x^{\prime} \int_{c}^{x^{\prime}}(.) \mathrm{d} x^{\prime \prime} .
\end{aligned}
$$

From (12), we note that $L_{x x}^{-1}\left(u^{\prime \prime}(x)\right)$ is already expressed in terms of the given boundary conditions without any restriction on $c$. Here, a question may be asked; how to choose the value of $c$ ? For answer, this choice depends properly on the singular point, if exists, of the equation under consideration. For example, if the equation has a singularity at $x=x_{0}$, say, we will choose $c$ to be any real value except the value of $x_{0}$. For example, consider the Thomas-Fermi equation [15] $u^{\prime \prime}(x)=x^{-1 / 2} u^{3 / 2}(x)$ subject to the boundary conditions $u(0)=1, u(1)=0$. It is easy to note that the inverse operator proposed by Lesnic and Elliott in [37] and given by (1) fails to overcome the singularity at $x=0$ for the Thomas-Fermi equation. However, the modified operator given by (5) can be used easily by choosing $c \neq 0$ to solve this problem. Moreover, if the equation has two singular points at $x=x_{1}$ and $x=x_{2}$, then we choose $c$ to be any real value except those of $x_{1}$ and $x_{2}$. In general, if the equation has $n$-singular points $x_{1}, x_{2}, \ldots, x_{n}$, then $c$ takes any real value except the values of these singular points. However, if the equation being solved has no singular points at all then we can use $c=0$, for simplicity. In the next section we use the proposed operator for solving two nonlinear boundary value problems: the nonlinear oscillator equation and Troesch's problem.

\section{Illustrative Examples}

\section{Example 1. Nonlinear Oscillator Equation}

Consider the nonlinear oscillator equation

$$
\begin{aligned}
& u^{\prime \prime}+\omega^{2} u=\lambda u^{m}, \\
& u(0)=0, \quad u(1)=\alpha, \quad \alpha>0 .
\end{aligned}
$$

This problem has the exact solution $u=\operatorname{sn}\left(x \mid \frac{1}{4}\right)$ when $m=3$ (Duffing oscillator), $\lambda=\frac{1}{2}, \omega^{2}=\frac{5}{4}$, and $\alpha=$ $\operatorname{sn}\left(1 \mid \frac{1}{4}\right)$ where sn is the Jacobi-elliptic function [16]. To solve (14), we rewrite the equation in the operator form:

$$
L_{x x}\left(u^{\prime \prime}(x)\right)=\lambda u^{m}-\omega^{2} u .
$$

Adomian's decomposition method (ADM) is based on decomposing $u$ and the nonlinear term $u^{m}$ as

$$
u=\sum_{n=0}^{\infty} u_{n} \quad \text { and } \quad u^{m}=\sum_{n=0}^{\infty} A_{n},
$$




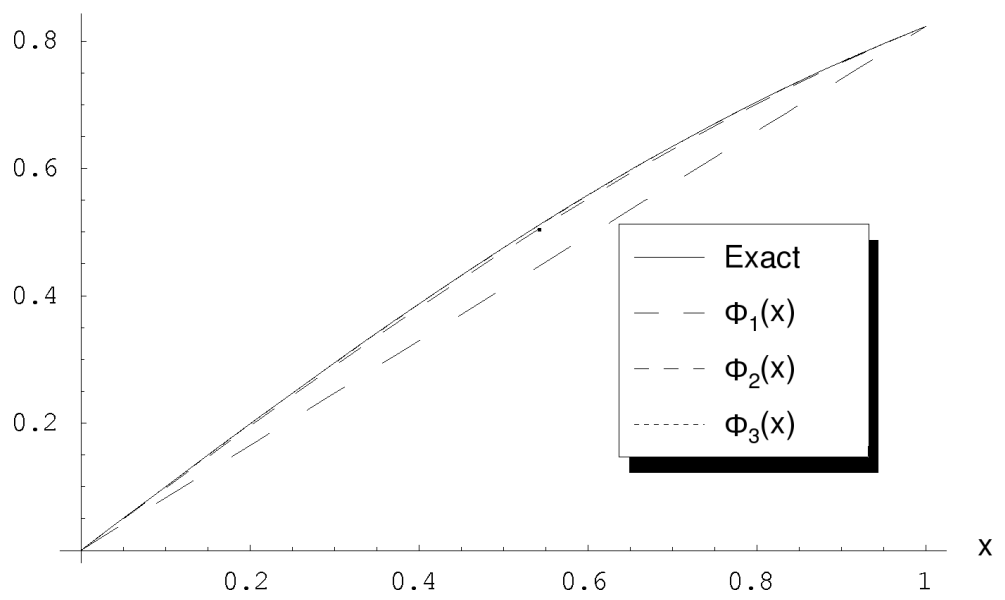

Fig. 1. Truncated ADM series solutions and the exact solution for Example 1.

where $A_{n}$ are Adomian's polynomials for the nonlinear term $u^{m}$ and can be found from the formula

$$
A_{n}=\frac{1}{n !}\left[\frac{\mathrm{d}^{n}}{\mathrm{~d} \lambda^{n}} N\left(\sum_{i=0}^{\infty} \lambda^{i} u_{i}\right)\right]_{\lambda=0}, \quad n \geq 0 .
$$

Now, operating with

$$
\begin{aligned}
& L_{x x}^{-1}(.)= \\
& \int_{0}^{x} \mathrm{~d} x^{\prime} \int_{c}^{x^{\prime}}(.) \mathrm{d} x^{\prime \prime}-x \int_{0}^{1} \mathrm{~d} x^{\prime} \int_{c}^{x^{\prime}}(.) \mathrm{d} x^{\prime \prime}, \quad c=0,
\end{aligned}
$$

on (15), then it follows

$$
u(x)=\alpha x+\lambda L_{x x}^{-1}\left(A_{n}\right)-\omega^{2} L_{x x}^{-1}(u) .
$$

According to the ADM, the solution can be elegantly computed by using the recurrence relation

$$
\begin{aligned}
& u_{0}=\alpha x \\
& u_{n+1}=L_{x x}^{-1}\left[\lambda A_{n}-\omega^{2} u_{n}\right], \quad n \geq 0 .
\end{aligned}
$$

The first few terms of Adomian's polynomials for the nonlinear term $u^{m}$ can be obtained from (17) as $A_{0}=u_{0}^{m}, A_{1}=m u_{0}^{m-1} u_{1}$, and $A_{2}=m u_{0}^{m-1} u_{2}+\frac{1}{2} m(m-$ 1) $u_{0}^{m-2} u_{1}^{2}$. Using the recurrence relation (20), we construct the $n$-term approximate solution as

$$
\Phi_{n}=\sum_{i=0}^{n-1} u_{i}
$$

For $m=3$, the first few components of the decomposi- tion series are given by

$$
\begin{aligned}
u_{0} & =\alpha x \\
u_{1} & =\frac{1}{60} \alpha x\left(10 \omega^{2}-3 \lambda \alpha^{2}\right)-\frac{1}{6} \alpha \omega^{2} x^{3}+\frac{1}{20} \lambda \alpha^{3} x^{5}, \\
u_{2} & =\frac{1}{50400} \alpha x\left(980 \omega^{4}+273 \lambda^{2} \alpha^{4}-1020 \lambda \alpha^{2} \omega^{2}\right) \\
& +\frac{1}{50400} \alpha x^{3}\left(-1400 \omega^{4}+420 \lambda \alpha^{2} \omega^{2}\right) \\
& +\frac{1}{50400} \alpha x^{5}\left(420 \omega^{4}-378 \lambda^{2} \alpha^{4}+1260 \lambda \alpha^{2} \omega^{2}\right) \\
& -\frac{11}{840} \lambda \alpha^{3} \omega^{2} x^{7}+\frac{1}{480} \lambda^{2} \alpha^{5} x^{9}
\end{aligned}
$$

For $\lambda=\frac{1}{2}, \omega^{2}=\frac{5}{4}$ and $\alpha=\operatorname{sn}\left(1 \mid \frac{1}{4}\right)$, the comparison of the approximate solutions $\Phi_{1}(x), \Phi_{2}(x)$, and $\Phi_{3}(x)$ and the exact one in Figure 1 shows a very good agreement using only three terms of the decomposition series solution. Furthermore, our approach is applied directly and easily without any need to Green's function [16].

\section{Example 2. Troesch's problem}

In this example we consider the boundary value problem, Troesch's problem [38-40],

$$
\begin{aligned}
& u^{\prime \prime}(x)=\lambda \sinh [\lambda u(x)], \quad 0 \leq x \leq 1, \\
& u(0)=0, \quad u(1)=1 .
\end{aligned}
$$

Troesch's problem arises from a system of nonlinear ordinary differential equations which occurs in an investigation of the confinement of a plasma column 
by radiation pressure. It was described and solved by Weibel [38]. Also authors of [39] solved it using Green's function with the Adomian decomposition method, and in [40] using the variational iteration method. To compare these approaches with the one developed in this paper, we apply the ADM as indicated above to obtain the following recurrence relation:

$$
\begin{aligned}
& u_{0}=x \\
& u_{n+1}=\lambda L_{x x}^{-1}\left[A_{n}\right], \quad n \geq 0 \\
& L_{x x}^{-1}(.)= \\
& \int_{0}^{x} \mathrm{~d} x^{\prime} \int_{c}^{x^{\prime}}(.) \mathrm{d} x^{\prime \prime}-x \int_{0}^{1} \mathrm{~d} x \int_{c}^{x^{\prime}}(.) \mathrm{d} x^{\prime \prime}, \\
& c=0 .
\end{aligned}
$$

The first few terms of Adomian's polynomials for the nonlinear term $\sinh (\lambda u(x))$ can be obtained from (17) as

$$
\begin{aligned}
& A_{0}=\sinh \left(\lambda u_{0}(x)\right) \\
& A_{1}=\lambda \cosh \left(\lambda u_{0}(x)\right) u_{1} \\
& A_{2}=\lambda \cosh \left(\lambda u_{0}(x)\right) u_{2}+\frac{1}{2} \lambda^{2} \sinh \left(\lambda u_{0}(x)\right) u_{1}^{2} .
\end{aligned}
$$

The first few components of the decomposition series are given by

$$
\begin{aligned}
& u_{0}=x, \\
& u_{1}=\frac{\sinh (\lambda x)-x \sinh (\lambda)}{\lambda},
\end{aligned}
$$

[1] G. Adomian, Solving frontier problems of physics: the decomposition method, Kluwer. Acad., Boston 1994.

[2] G. Adomian, Appl. Math. Comput. 88, 117 (1997).

[3] G. Adomian, Appl. Math. Lett. 11, 121 (1998).

[4] Y. Cherruault and K. Abbaoui, Math. Comput. Modelling. 24, 89 (1996).

[5] A. Ebaid, Comput. Appl. Math. 223, 278 (2009).

[6] D. Kaya and S. M. El-Sayed, Phys. Lett. A 318, 345 (2003).

[7] J. Biazar, M. Ilie, and A. Khoshkenar, Appl. Math. Comput. 171, 486 (2005).

[8] I. Hashim, M. S. M. Noorani, R. Ahmad, S. A. Bakar, E. S. Ismail, and A.M. Zakaria, Chaos, Solitons, and Fractals 28, 1149 (2006).

[9] D. Lesnic, Chaos, Solitons, and Fractals 28, 776 (2006).

[10] Y. Cherruault, Kybernetes 9, 31 (1988).

$$
\begin{aligned}
& u_{2}=\frac{1}{4 \lambda^{2}}[3 \lambda x \sinh (\lambda) \cosh (\lambda) \\
& -4 \lambda x \sinh (\lambda) \cosh (\lambda x)-8 x \sinh ^{2}(\lambda) \\
& +8 \sinh (\lambda) \sinh (\lambda x)+\lambda \sinh (\lambda x) \cosh (\lambda x)]
\end{aligned}
$$

Also, these results are in full agreement with those obtained in [39] by Green's function with ADM. However, our proposed approach is used easily and directly without any need to Green's function again.

\section{Conclusions}

In this paper, we proposed an efficient approach for solving nonlinear Dirichlet boundary value problems (BVPs) of second order. The proposed approach is used in a direct way if compared with the previous works. This modification has been applied successfully for solving two physical nonlinear non-singular boundary value problems. Also, for regular-singular two-point BVPs we have shown in [41] that the proposed modification is still effective. However, this approach may have some disadvantages when applied to second-order BVPs with irregular singularities. So, further modifications are needed to achieve this goal and it is our objective in the present time.

\section{Acknowledgements}

The author would like to thank the referees for their comments and discussions.

[11] Y. Cherruault, G. Saccamondi, and B. Some, Math. Comput. Modell. 16, 85 (1992).

[12] Y. Cherruault and G. Adomian, Math. Comput. Modell. 18, 103 (1993).

[13] Y. Cherruault, G. Adomian, K. Abbaoui, and R. Rach, Int. J. Bio-Medical Comput. 38, 89 (1995).

[14] G. Adomian and R. Rach, J. Math. Anal. Appl. 174, 118 (1993).

[15] G. Adomian, Appl. Math. Lett. 11, 131 (1998).

[16] N. T. Shawagfeh, Comput. Math. Appl. 31, 135 (1996).

[17] M. Hadizadeh and K. Maleknejad, Kybernetes 27, 426 (1998).

[18] N. T. Eldabe, E. M. Elghazy, and A. Ebaid, Phys. Lett. A 363, 257 (2007).

[19] A. Ebaid, Z. Naturforsch. 65a, 145 (2010).

[20] M. Benabidallah and Y. Cherruault, Kybernetes 33, 118 (2004). 
[21] M. Benabidallah and Y. Cherruault, Kybernetes 33, 1185 (2004).

[22] M. Benabidallah and Y. Cherruault Kybernetes 33, 1292 (2004).

[23] W. Al-Hayanil and L. Casasus, Comput. Appl. Math. 177, 187 (2005).

[24] A.-M. Wazwaz, Comput. Appl. Math. 136, 259 (2001).

[25] A.-M. Wazwaz, Found. Phys. Lett. 13, 493 (2000).

[26] I. Hashim, Comput. Appl. Math. 193, 658 (2006).

[27] M. Dehghan, Appl. Math. Comput. 157, 549 (2004).

[28] M. Dehghan and R. Salehi, Commun. Numer. Meth. Eng. In Press. DOI: 10.1002/cnm.1315.

[29] M. Dehghan and R. Salehi, Numer. Meth. Partial Diff. Eqs. In Press. DOI: 10.1002/num.20482.

[30] M. Dehghan and F. Shakeri, Physica Scripta 78 2008, Article No. 065004, (11 pages).

[31] M. Dehghan and F. Shakeri, Numer. Meth. Partial Diff. Eqs. 25, 1238 (2009).

[32] M. Tatari, M. Dehghan, and M. Razzaghi, Math. Comput. Modell. 45, 639 (2007).
[33] M. Tatari and M. Dehghan, Physica Scripta 72, 345 (2005).

[34] M. Dehghan and M. Tatari, Math. Prob. Eng. 2006, 1 (2006).

[35] M. Dehghan, M. Shakourifar, and A. Hamidi, Chaos, Solitons, and Fractals 39, 2509 (2009).

[36] M. Dehghan, A. Hamidi, and M. Shakourifar, Appl. Math. Comput. 189, 1034 (2007).

[37] D. Lesnic and L. Elliott, J. Math. Analys. Appl. 232, 82 (1999).

[38] E. S. Weibel, in: The Plasma in Magnetic Field, (Ed. R. K. M. Landshoff), Stanford University Press, Stanford 1958.

[39] E. Deeba and S. A. Khuri, J. Comput. Phys. 159, 125 (2000).

[40] S. Momani, S. Abuasad, and Z. Odibat, Appl. Math. Comput. 183, 1351 (2006).

[41] A. Ebaid, Comput. Appl. Math., submitted. 